\title{
Application of Virtual Simulation in Diversified Pharmacy Teaching Mode
}

\author{
Weifeng Peng
}

Zhoukou Normal University, Zhoukou 466001, Henan, China. E-mail: pengwf226@163.com

Abstract: In the course of pharmacy course study, experimental teaching is an important way to encourage students to effectively combine theoretical knowledge and practical application, and it occupies a very important position in actual teaching. This article combines the actual situation of pharmaceutics teaching, analyzes the current status of pharmaceutics experimental teaching, and discusses the feasibility of virtual simulation technology in pharmaceutics experimental teaching, hoping that this technology can be used to increase students' interest in learning and stimulate students' learning Enthusiasm, reduce the cost and risk of experimental teaching, and provide some new thinking for the reform of pharmacy experimental teaching.

Keywords: Pharmaceutics; Virtual Simulation Technology; Teaching Mode; Applied Research

From the actual teaching effect, virtual simulation technology can effectively circumvent various problems in pharmacy experimental teaching, so that students can have a better classroom experience, and the teaching efficiency of teachers has also been greatly improved. Combining many years of pharmacy teaching practice, the author analyzes the application of virtual simulation technology in experimental teaching from multiple angles, hoping to contribute to the promotion of virtual simulation teaching methods.

\section{The current situation of pharmaceutical experiment teaching}

First, the faculty and hardware facilities of universities are limited. Limited by the school's basic experimental conditions and the teacher's arrangement of teaching courses, traditional pharmacy experimental teaching is basically based on confirmatory experiments. Students only verify textbook experiments and do not further in-depth experiments on this basis. The content itself has not achieved the expected results in the cultivation of students' innovative ability. Secondly, the experimental projects are basically based on traditional medicines, and the latest pharmaceutics achievements are not or rarely involved, and students' knowledge of pharmacy is limited. Finally, the experimental funds of universities are limited, and basically all need to accept the budget management of the school. The experimental instruments are basically expensive, and there is no guarantee that every student has enough time for practical operations. In addition, no matter what kind of experiment, there are certain safety hazards. Therefore, in order to protect the safety of students, experimental teaching rarely involves those experiments with high cost, high risk factor, and long operation time. The above three points are basically the important reasons that limit the current development of pharmaceutics experimental teaching.

\section{Feasibility study on application of virtual simulation technology in diversified pharmacy teaching}

\subsection{Break through many teaching restrictions and improve teaching effects}

Traditional pharmaceutics experimental teaching will be subject to restrictions such as time, location, experimental environment, experimental materials, etc., and relevant preparations must be made before the experiment. Experiments cannot be carried out without any of them. The virtual simulation technology can break the limitations of the original experimental

Copyright(C) 2020 Weifeng Peng

doi: 10.18686/ahe.v4i10.2909

This is an open-access article distributed under the terms of the Creative Commons Attribution Non-Commercial License (http://creativecommons. org/licenses/by-nc/4.0/), which permits unrestricted non-commercial use, distribution, and reproduction in any medium, provided the original work is properly cited. 
conditions, only need to input all the experimental conditions data into the computer to perform the experimental simulation. In addition, the application of virtual simulation technology can eliminate the limitation of the number of students and experimental equipment, allowing students to conduct simulation experiments at will. Teachers can also set up various test sites in simulation teaching according to the specific conditions of the experiment, which greatly improves experimental teaching. Effectiveness.

\subsection{Greatly enriched experimental projects}

From the actual teaching situation, in pharmacy experimental teaching, teachers are limited by experimental equipment and other factors. They basically carry out traditional pharmacy experimental projects. The experimental content is single and fixed, and with the development of the times, the traditional experimental content Presents a kind of lag, which is very unfavorable to students' learning. The application of virtual simulation technology can broaden the teaching thinking of teachers, consider the loss of equipment and medicament in disorder, apply more advanced experimental teaching, and set up some experimental teaching close to actual production, so that students can familiarize themselves with various experiments. The operating specifications and corresponding experimental essentials have effectively saved laboratory operating costs, enriched experimental teaching projects, and allowed students to deeply appreciate the charm of pharmacy experiments.

\subsection{Avoid experimental risks, and no need to deal with experimental waste}

Pharmacy experiments are similar to chemical experiments. Both require exposure to certain hazardous chemicals, and the experimental waste generated after the experiment needs to be treated specifically to prevent adverse effects on the surrounding environment, which virtually improves the experimental teaching Risk and cost are also one of the important factors restricting the experimental teaching of traditional pharmacy. The application of virtual simulation technology can effectively avoid these problems. Because it is a simulation experiment, there is no need to worry about the safety hazards caused by the experimental materials, and there is no need to worry about the safe disposal of waste after the experiment, allowing teachers to open more high-risk important experimental projects, Allowing students to experience the serious consequences of experimental errors in this process, improve students' awareness of crisis, and truly realize "green" teaching.

\subsection{Can provide more timely feedback on experimental results and improve the effectiveness of experimental teaching}

In traditional experimental teaching, students cannot accurately control every step of the experiment operation, and a lot of practice is needed to ensure the accuracy of the experiment. The application of virtual simulation technology can greatly increase the speed of this process. The virtual simulation experiment platform can input the standardized experimental process in the early stage of the experiment. Students can clearly know whether each step and the operating standard are different during the virtual operation, and can suspend the experiment at any time when there is a problem. Therefore, the accuracy and standardization of students' experiments can be quickly improved, which greatly enhances students' self-confidence in learning, and deepens students' understanding of experimental operations.

\section{Conclusion}

To sum up, the application of virtual simulation technology has a great promotion effect on the experimental teaching of pharmacy, and it is an information teaching method that conforms to the development of the current era. Teachers can get rid of the various limitations of traditional experimental teaching, and are no longer restricted by time and space, personnel, and experimental equipment. This improves the safety of experiments, reduces the loss of experiments, enriches experimental projects, and expands students' knowledge. For students, virtual simulation technology can greatly enhance students' experimental teaching experience, enhance their enthusiasm for learning, and allow students to adapt to various experimental operations faster, which has a very positive effect on improving their professional capabilities.

\section{References}

1. Li WL, Li X, Yang B. Application of virtual simulation technology in pharmaceutics teaching. Guangzhou Chemical Industry 2019; (07): 25-26.

2. Wu C. Research on the application of diversified teaching mode in pharmaceutics practice teaching. Health Vocational Education 2018; 36(19): 111-113.

3. Zhu WQ, Han CY, Dong Y, et al. Application of virtual simulation training teaching method in pharmaceutics training teaching. Continuing Medical Education 2018; 032(004): 51-52. 